\title{
Supporting Information: \\ Tuning the Structure and Properties of Ultra-Low Cross-Linked Temperature-Sensitive Microgels at Interfaces via the Adsorption Pathway
}

\author{
M. Friederike Schulte, ${ }^{1,2}$ Andrea Scotti, ${ }^{1}$ Monia Brugnoni, ${ }^{1}$ \\ Steffen Bochenek, ${ }^{1}$ Ahmed Mourran, ${ }^{2}$ and Walter Richtering*1,2 \\ ${ }^{1}$ Institute of Physical Chemistry, RWTH Aachen University, Landoltweg 2, 52056 Aachen, Germany \\ ${ }^{2}$ DWI - Leibniz Institute for Interactive Materials, Forckenbeckstr. 50, 52056 Aachen, Germany
}

Corresponding author: *E-mail: richtering@rwth-aachen.de

\section{CONTENTS}

1. List of abbreviations 2

2. Sample code 3

3. Static Light Scattering (SLS) and Small-Angle Neutron Scattering (SANS) 3

4. AFM images at the solid/air interface 3

5. AFM images after in situ adsorption and drying 4

6. Details of the experimental procedure of the AFM measurements at the solid/liquid interface 5

7. AFM image analysis 6

7.1. AFM phase image analysis for extraction of contact radii of dry microgels 6

7.2. AFM analysis for extraction of radial height profiles 6

7.3. AFM image analysis for volume calculation of adsorbed microgels 6

7.4. AFM image analysis for determination of the nearest-neighbor distance $\quad 7$

8. AFM height images of ULC microgels deposited from the oil/water interface by the Langmuir-Blodgett technique

9. Volume calculation of adsorbed microgels $\quad 8$

10. Impact of applied force during imaging $\quad 9$

$\begin{array}{ll}\text { References } & 9\end{array}$ 


\section{LIST OF ABBREVIATIONS}

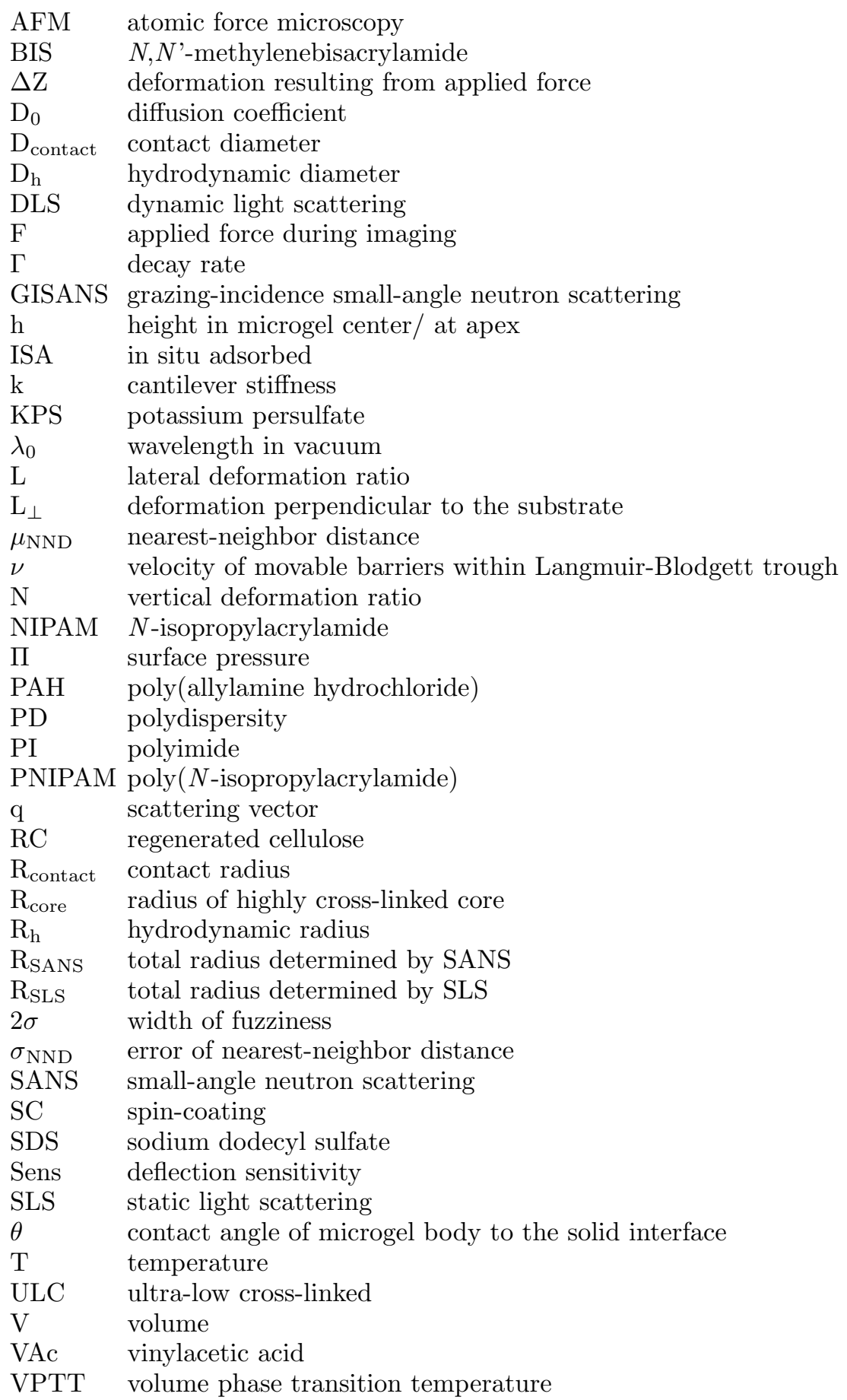




\section{SAMPLE CODE}

The $5 \mathrm{~mol} \%$ BIS microgels were synthesized by Monia Brugnoni and have the lab journal code MB-pNIPAM5mol\%BIS-225nm/SFB985_A3_MB_M000186. The ultra-low cross-linked (ULC) microgels were synthesized by Andrea Scotti and have the lab journal code SCOT1-ULC/SFB985_A3_SCO_M000508.

\section{STATIC LIGHT SCATTERING (SLS) AND SMALL-ANGLE NEUTRON SCATTERING (SANS)}

The internal structure of $5 \mathrm{~mol} \%$ BIS (A, filled circles) and ULC microgels (B, open circles) in dilute solution was determined by form factor analysis (Figure $\mathbf{S 1}$ ).
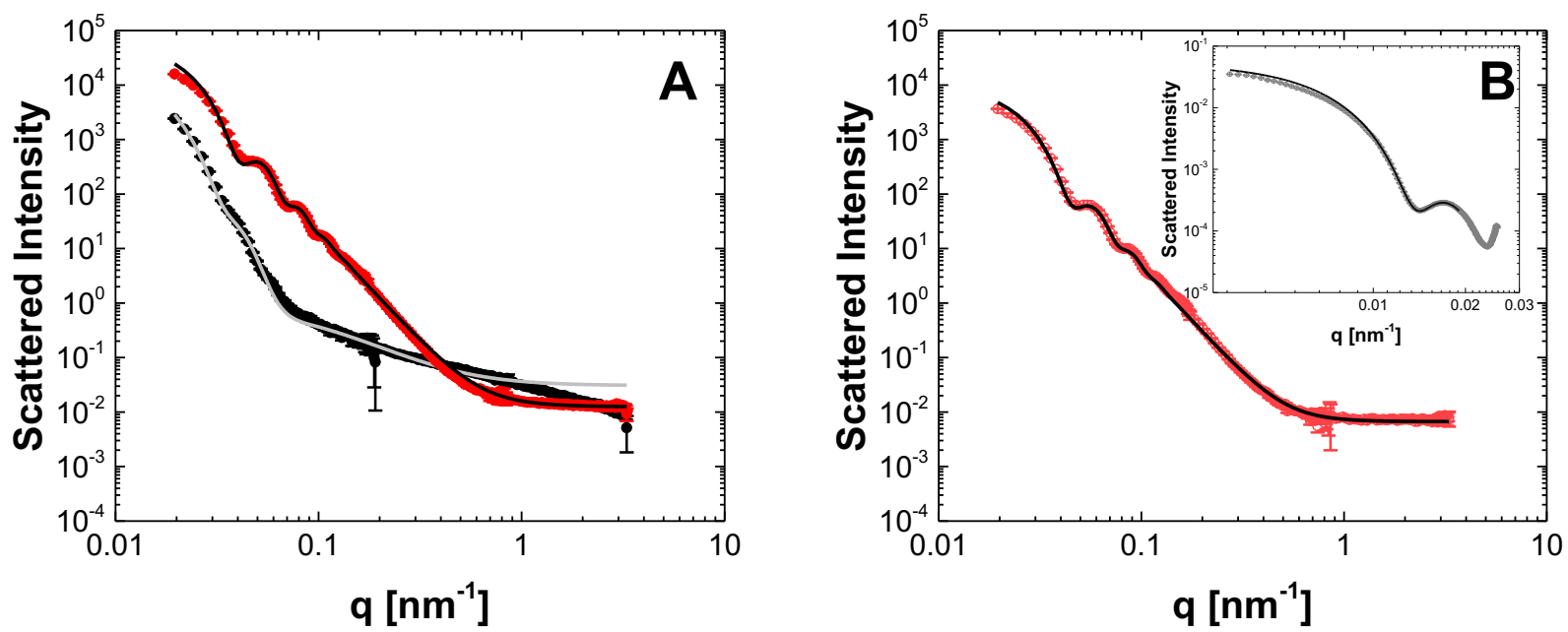

Figure S1. Form factors probed by SANS of $5 \mathrm{~mol} \%$ BIS (A, filled circles) and ULC (B, open circles) microgels at $T=20^{\circ} \mathrm{C}$ (black/gray) and $T=40^{\circ} \mathrm{C}$ (red/light red). The data were fitted with the fuzzy-sphere model[1] and are represented by the solid lines (gray/ black). Inset in B: Form factor of ULC microgels at $T=20^{\circ} \mathrm{C}$ probed by SLS as they are too large to be analyzed by SANS.

The scattered intensities from small-angle neutron scattering and static light scattering experiments were measured in dependence of the scattering vector $q$ in the swollen state, at $T=20^{\circ} \mathrm{C}$ (black/ gray), and in collapsed state, at $T=40^{\circ} \mathrm{C}$ (red/ light red) for both microgel species. The data were fitted with the fuzzy-sphere model [1] and the fits are depicted by the solid lines. The relative polymer volume fraction as a function of the radial position within the microgel were determined by the fits and are shown in Figure $\mathbf{1 B}$ and $\mathbf{C}$.

\section{AFM IMAGES AT THE SOLID/AIR INTERFACE}

$5 \mathrm{~mol} \%$ BIS and ULC microgels were deposited onto PAH-coated glass substrates via spin-coating of microgel solutions at $T<$ VPTT. The adsorbed microgels in the dry state were ex situ investigated by AFM in tapping mode (Figure S2).

Figure S2A shows the AFM height images of the $5 \mathrm{~mol} \%$ BIS microgels in dry state. The microgels exhibit a typical 'fried-egg' shape, with a densely cross-linked center with a maximum height of 63 nm surrounded by a less cross-linked and flat corona $(1-2 \mathrm{~nm}$ in height). In the corresponding phase image (Figure S2B), this distinction into highly and loosely cross-linked area gets very clear. Additionally, a dark brown contrast around the individual microgels is visible, resulting from the microgel-uncovered substrate, showing that the microgels are separated from each other.

The height image of the ULC microgels in dry state is shown in Figure S2C. In contrast to the 5 mol\% BIS microgels, the ULC microgels have a very different structure. They are forming very flat and homogenous layers with a thickness of ca. $1.5 \mathrm{~nm}$. This 'pancake-like structure is also observed in the corresponding phase image (Figure S2D).

The differences of the structures in the dry state for the two microgel species are a result of their different internal structure in bulk solution in the swollen state. Further analysis of the images can be found in the description of Figure 3. 

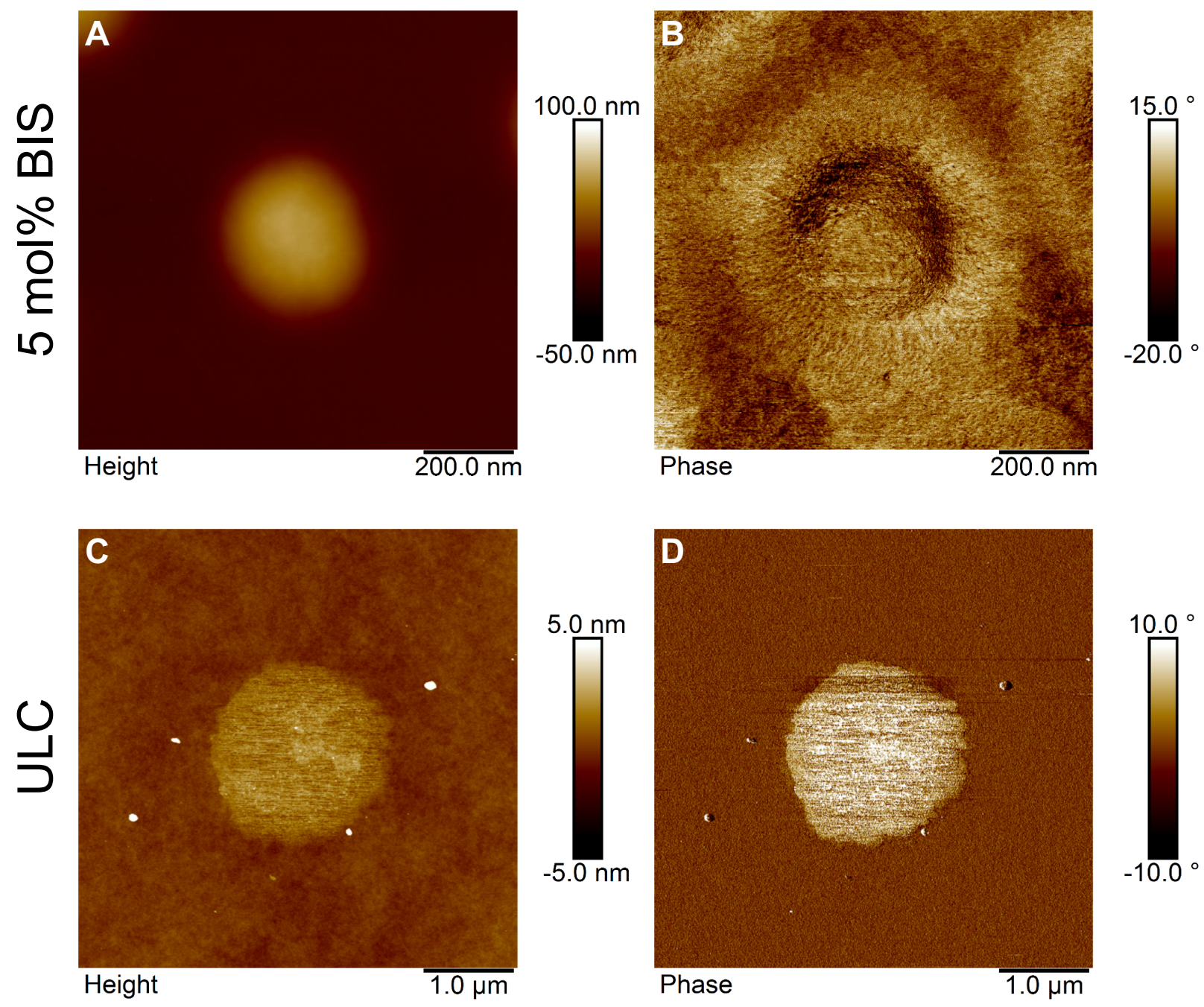

Figure S2. AFM images of $5 \mathrm{~mol} \%$ BIS $(\mathbf{A}+\mathbf{B})$, and ULC microgels $(\mathbf{C}+\mathbf{D})$ deposited via spin-coating onto PAH-coated glass substrates. The microgels were spin-coated from solution at $T<$ VPTT and were imaged in dry state by tapping mode measurements. The AFM images are divided in height $(\mathbf{A}+\mathbf{C})$ and the corresponding phase images $(\mathbf{B}+\mathbf{D})$. Attention should be paid to the different scales of the images for the different microgel species.

\section{AFM IMAGES AFTER IN SITU ADSORPTION AND DRYING}

ULC microgels were deposited onto PAH-coated glass substrates via in situ adsorption. The interface was dried after the analysis of the collapsed state and investigated by AFM in tapping mode in the dry state (Figure S3).

Drying of the interface led to an accumulation of the dirt previously adsorbed to the liquid/air interface. The contamination could not be prevented even by several washing attempts of the solid interface. No protruding structures are visible in the height image except for the dirty areas. However, a light brown contrast is observed in the corresponding phase image. The area of this contrast has a similar contact radius to the solid substrate as the ULC microgels in dry state after spin-coating. It suggests, that these areas result from dry ULC microgels and that the drying process causes a strong deformation of the microgels, independent on the previous adsorption pathway. Since the resolution of the height and phase images in the dry state after in situ adsorption is not sufficient (due to the contamination with dirt), the images are not analyzed in a quantitative manner. 

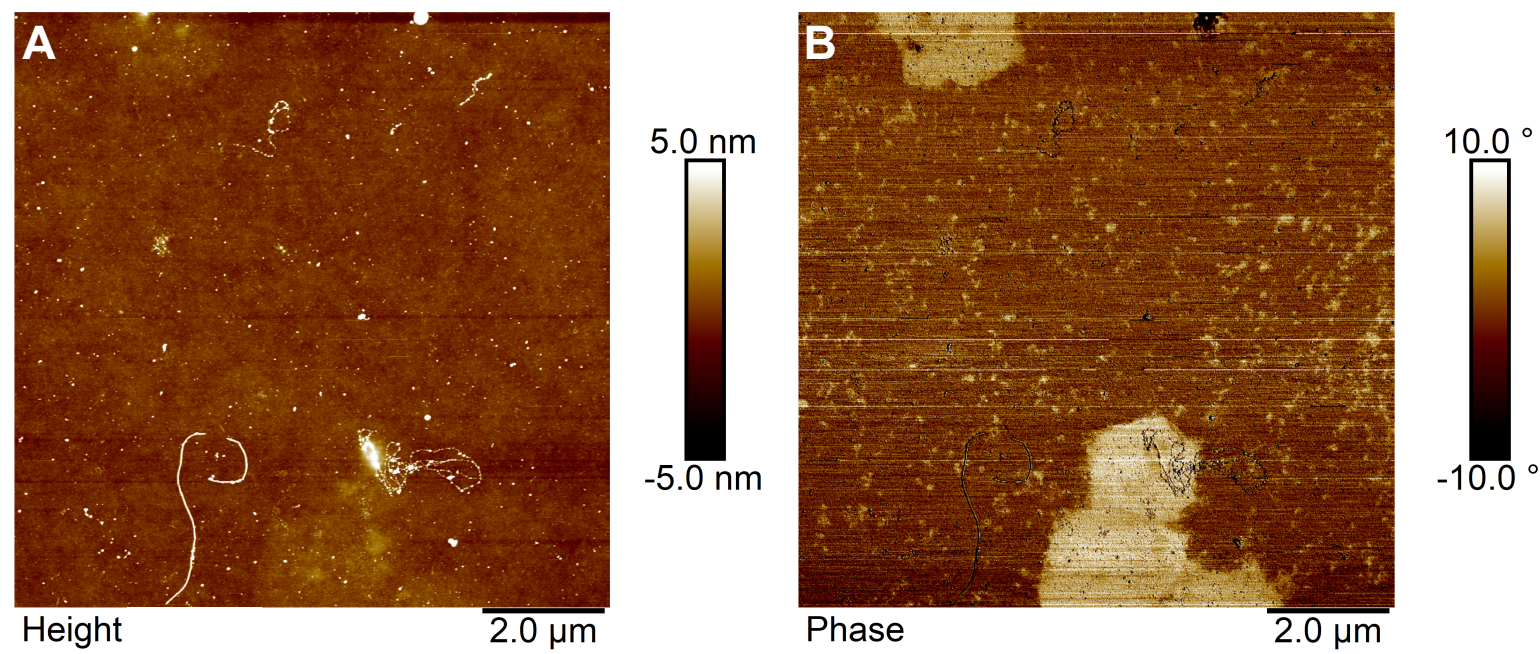

Figure S3. AFM height (A) and corresponding phase (B) images of ULC microgels after in situ adsorption onto a PAH-coated glass substrate and drying. The microgels in dry state were imaged by tapping mode measurements. Accumulation of dirt at the dry interface can be observed.

\section{DETAILS OF THE EXPERIMENTAL PROCEDURE OF THE AFM MEASUREMENTS AT THE SOLID/LIQUID INTERFACE}

The measurements were performed on a Dimension Icon AFM with closed loop (Veeco Instruments Inc., software: Nanoscope 9.4 (Bruker Corporation)). They were analyzed by the Nanoscope Analysis 1.9 Software (Bruker Corporation).

The investigations at the solid/liquid interface were conducted in a customized liquid cell on a heating stage (Dimension Icon Electrochemistry Chuck, Bruker Corporation) with temperature control (Model 335 Cryogenic Temperature Controller, Lake Shore Cryotronics). The glass substrate (with deposited microgels via spin-coating and LangmuirBlodgett technique on it or the PAH-coated substrate before in situ adsorption of microgels) was placed in the liquid cell, with a $55 \mu \mathrm{m}$ thick polyimide (PI)-foil beneath at the detector side. The microgels adsorbed via spin-coating and Langmuir-Blodgett technique were rehydrated by adding filtered $(0.2 \mu \mathrm{m} \mathrm{RC})$, not pre-heated, double-distilled water at a controlled temperature of $T=35^{\circ} \mathrm{C}$ of the liquid cell and the microgels adsorbed in situ were still hydrated as they were kept under water after adsorption. In total the microgels were kept at least two days in the hydrated state and heating/cooling cycles were conducted, showing a reversibility of the VPTT of the microgels at the interface. The experiments were performed at $T=35^{\circ} \mathrm{C}$. The temperature was equilibrated for ca. 60 minutes. The measurements were recorded in the Peak Force QNM mode with modified MSNL (Bruker Corporation) tips. AFM tips were activated by oxygen plasma treatment (in a PVA TePla plasma system 100) under 1.2 mbar oxygen pressure at $200 \mathrm{~W}$ of microwave power for $5 \mathrm{~min}$. Subsequently, the tip was used for imaging. Specifically the D and E probes were used with a nominal resonance frequency of 15 and $38 \mathrm{kHz}$ in air and a nominal spring constant of 0.03 and $0.1 \mathrm{~N} / \mathrm{m}$ of the cantilever, respectively (tip radius: $2 \mathrm{~nm}$, semi angle of the tip: $23^{\circ}$, assumed sample Possions ratio: 0.3 ). Before images were taken, the tips used were calibrated. For that reason, force-spectroscopy measurements on the solid substrate were conducted. Out of the slope of the deflection-distance-curves (after the contact-point), the cantilever deflection sensitivity was determined. The tip was withdrawn from the surface by $1000 \mu \mathrm{m}$ and the thermal noise was measured by the thermal tune of the Nanoscope 9.4 software. The temperature and the average displacement of the cantilever due to thermal noise give rise to the cantilever spring constant. The determined sensitivities Sens and spring constants $k$ of the used tips are listed in Table S1. During imaging, the integrated ScanAsyst was used to control the feedback in terms of Z limit. Peak Force Amplitude, Peak Force Frequency, Peak Force Setpoint, and Peak Force Gain were set manually. The used scan parameters are listed in Table $\mathbf{S} \mathbf{1}$ for the different measurements. 
Table S1. Characteristics of the tips and Peak Force scan parameter used for measurements of the adsorbed microgels at the solid/liquid interface. The $5 \mathrm{~mol} \%$ BIS and ULC microgels were deposited i) via in situ adsorption, ii) spin-coating, or iii) Langmuir-Blodgett (LB) deposition. Tip characteristics include the MSNL (Bruker Corporation) probe type, deflection sensitivity Sens, and cantilever spring constant $k$. The Peak Force scan parameter are specified in terms of Peak Force Amplitude, Frequency, Setpoint, and Gain. FB: scan parameter was feedback controlled by the integrated ScanAsyst.

\begin{tabular}{|c|c|c|c|c|c|c|c|c|}
\hline \multirow[b]{2}{*}{ Sample type } & & \multicolumn{3}{|c|}{ Tip characteristics } & \multicolumn{4}{|c|}{ Peak Force scan parameter } \\
\hline & & type & $\begin{array}{c}\text { Sens } \\
{[\mathrm{nm} / \mathrm{V}]}\end{array}$ & $\begin{array}{c}\mathrm{k} \\
{[\mathrm{N} / \mathrm{m}]} \\
\end{array}$ & $\begin{array}{c}\text { Amplitude } \\
{[\mathrm{nm}]}\end{array}$ & $\begin{array}{c}\text { Frequency } \\
{[\mathrm{kHz}]}\end{array}$ & $\begin{array}{c}\text { Setpoint } \\
{[\mathrm{nN}]}\end{array}$ & Gain \\
\hline \multirow{2}{*}{$5 \mathrm{~mol} \% \mathrm{BIS}$} & adsorbed in situ & $\mathrm{E}$ & 68.60 & 0.11 & 200 & 1.0 & 1.0 & 10.0 \\
\hline & spin-coated & $\mathrm{E}$ & 72.29 & 0.12 & 200 & 2.0 & 1.0 & 10.0 \\
\hline \multirow{3}{*}{ ULC } & adsorbed in situ & $\mathrm{D}$ & 106.7 & 0.04 & 200 & 0.25 & 0.5 & 3.00 \\
\hline & spin-coated & $\mathrm{E}$ & 83.38 & 0.10 & 160 & 2.0 & 1.2 & $\mathrm{FB}$ \\
\hline & LB deposition & $\mathrm{E}$ & 63.52 & 0.12 & 200 & 1.0 & 1.0 & FB \\
\hline
\end{tabular}

\section{AFM IMAGE ANALYSIS}

For all analyses of the AFM images only microgels which had sufficient spacing from neighboring microgels were taken into account to eliminate effects of dense packing on the results.

\subsection{AFM phase image analysis for extraction of contact radii of dry microgels}

AFM phase images of the dry microgels were analyzed with the public domain software ImageJ $1.47 \mathrm{v}$ to determine the total contact radii to the solid substrate. The perimeter of circles around the contrast resulting from the microgels was measured for at least 65 microgels of each type and averaged. The corresponding radius and its deviation were determined and are listed in Table $\mathbf{1}$.

\subsection{AFM analysis for extraction of radial height profiles}

Height images were analyzed by a MATLAB routine (MathWorks, 2016a).[2] The center of the microgels was determined by this routine. The microgel was partitioned into 100 ellipsoids with increasing radius around the center. The height was averaged for every ellipsoid. Height as a function of the radial position were received. The radial profiles were averaged for all microgels of the same species. Due to the special morphology of the ULC microgels in collapsed state deposited via spin-coating, the MATLAB routine could not be used. Representative height cross sections are shown instead (see Figure $4 \mathbf{B}$ ) and the contact radius to the substrate $R_{\text {contact, } 35^{\circ} \mathrm{C}, \mathrm{SC}}$ was determined with the public domain software Image J $1.47 \mathrm{v}$ similarly as above, but for the AFM height images.

\subsection{AFM image analysis for volume calculation of adsorbed microgels}

Height images of the microgels were analyzed by a MATLAB routine (MathWorks, 2016a).[2] The microgels were distinguished from the background resulting from the solid substrate. The height information of every pixel was multiplied by the pixel area. The resulting product was summed up for all pixels belonging to one microgel to receive the volume of the adsorbed microgel. The volumes were averaged for all microgels of the same species. In case of the dry $5 \mathrm{~mol} \%$ BIS microgels, a volume of a cylinder with the total contact radius determined from the phase images as described above and a height of $1 \mathrm{~nm}$ for the polymeric thin layer around the protruding densely cross-linked part was added to the volume determined by the MATLAB routine from the height images. In case of the dry ULC microgels, the volume of a cylinder with the total contact radius and a height of $1.5 \mathrm{~nm}$ was calculated. The determined volumes are listed in Table $\mathbf{S 2}$ and $\mathbf{S 3 .}$ 


\subsection{AFM image analysis for determination of the nearest-neighbor distance}

Height images of the ULC microgels deposited via Langmuir-Blodgett technique from the oil/water interface were analyzed with a custom-written MATLAB script (MathWorks, 2019a). The centers of the microgels were determined with the public available MATLAB version of the IDL particle tracking code by Crocker and Grier.[3] A Delaunay triangulation was used to compute the nearest-neighbor connections of the centers. The nearest-neighbor distance $\mu_{N N D}$ was determined by a single Gaussian fit to the distance probability function of the Delaunay triangulation and the variance is considered as the error $\left(\sigma_{N N D}\right)$.

\section{AFM HEIGHT IMAGES OF ULC MICROGELS DEPOSITED FROM THE OIL/WATER INTERFACE BY THE LANGMUIR-BLODGETT TECHNIQUE}
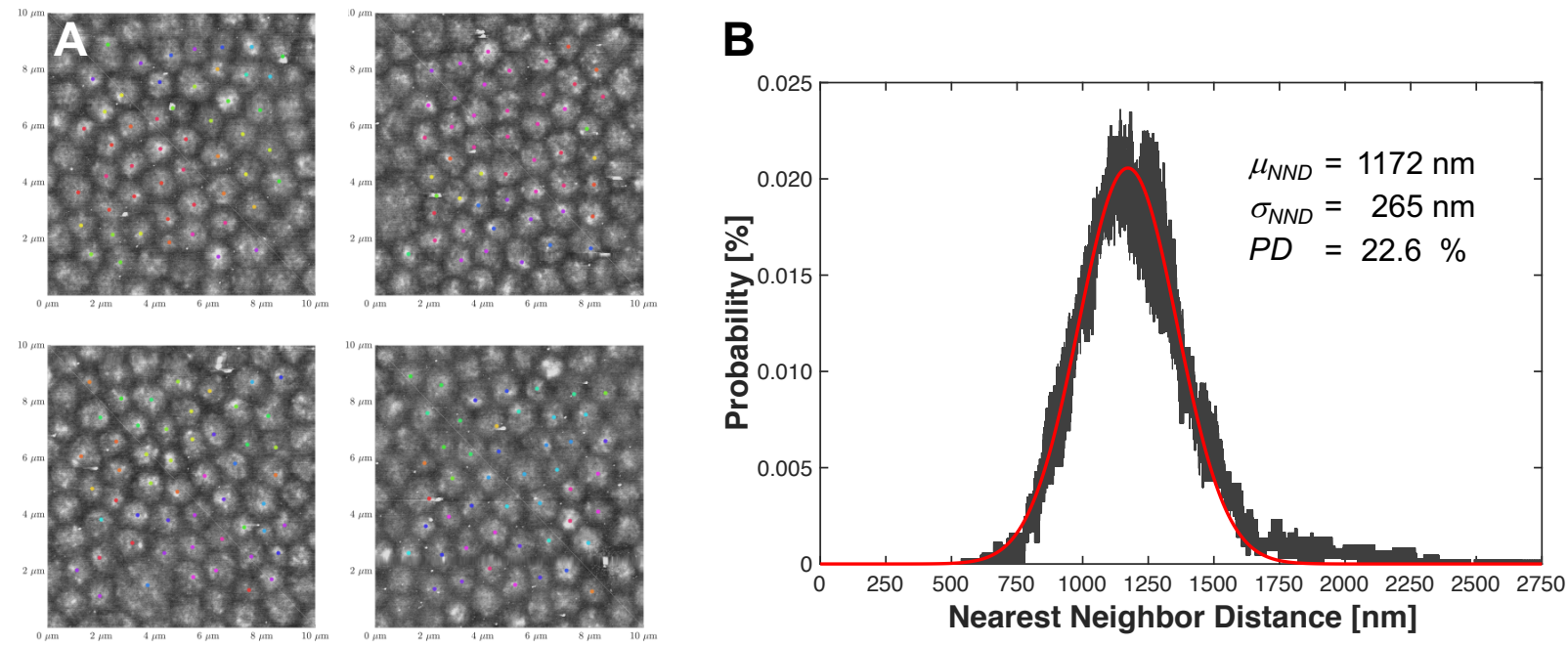

Figure S4. A: AFM height images of ULC microgels deposited from an oil/water interface on a PAH-coated glass substrate with a Langmuir-Blodgett trough at a constant surface pressure of $\Pi=29.3 \mathrm{mN} / \mathrm{m}$. The images are obtained by tapping mode measurements in dry state. The center of the microgels were determined via a MATLAB routine modified by Bochenek (see Experimental Section) and are marked. B: The corresponding distribution function of the nearest-neighbor distance $\mu_{N N D}$ (with error $\sigma_{N N D}$ and polydispersity $P D=\mu_{N N D} / \sigma_{N N D}$ ).

ULC microgels were deposited via the Langmuir-Blodgett technique from the oil/water interface. The microgels adsorbed to a water/decane interface within the Langmuir-Blodgett trough at $T<$ VPTT. After equilibration, the surface pressure was increased to $\Pi=29.3 \mathrm{mN} / \mathrm{m}$ and kept constant during the deposition of the microgel monolayer onto the PAH-coated glass substrate. Figure $\mathbf{S} 4$ shows the AFM height images obtained ex situ in the dry state. The solid support is fully covered by the ULC microgels. The center of the individual microgels was determined by a MATLAB routine modified by Bochenek (see above, subsection 6.4.) and the nearest-neighbor distance was extracted. The nearest-neighbor distance is $\mu_{N N D}=1172 \pm 265$ (polydispersity of 22.6\%). 


\section{VOLUME CALCULATION OF ADSORBED MICROGELS}

The volumes of the $5 \mathrm{~mol} \%$ BIS and ULC microgels adsorbed to a PAH-coated substrate via in situ adsorption and studied in the collapsed state $\left(T=35^{\circ} \mathrm{C}\right)$ were determined as described in the Experimental Section and are listed in Table S2. In addition they were compared to the microgels volume in bulk solution in the collapsed state, as well. $5 \mathrm{~mol} \%$ BIS microgels exhibit nearly the same volume at the interface as in bulk, whereas the volume is reduced to only $60 \%$ at the interface related to the one in bulk in case of the ULC microgels. Further analysis can be found in the discussion of Figure 2.

Table S2. Volumes obtained by AFM height images $V_{A F M}$ of the microgels adsorbed to a PAH-coated glass substrate via in situ adsorption, volumes obtained by DLS $V_{D L S}$, and ratio between the two $V_{A F M} / V_{D L S}$. Volumes $V_{A F M}$ were extracted as described in the Experimental Section. Volumes $V_{D L S}$ of the microgels in bulk were calculated using hydrodynamic radii $R_{h}$ at $T=40^{\circ} \mathrm{C}$ as a radius of a sphere.

\begin{tabular}{lccc}
\hline Sample type & $\begin{array}{c}\mathbf{V}_{\mathbf{A F M}, \mathbf{3 5}^{\circ} \mathbf{C}} \\
{\left[\mathbf{n m}^{\mathbf{3}}\right]}\end{array}$ & $\begin{array}{c}\mathbf{V}_{\mathbf{D L S}, \mathbf{4 0}}{ }^{\circ} \mathbf{C} \\
{\left[\mathbf{n m}^{\mathbf{3}}\right]}\end{array}$ & $\begin{array}{c}\mathbf{V}_{\mathbf{A F M}, \mathbf{3 5}^{\circ} \mathbf{C}} / \mathbf{V}_{\mathbf{D L S}, \mathbf{4 0}^{\circ} \mathbf{C}} \\
{[\%]}\end{array}$ \\
\hline $5 \mathrm{~mol} \%$ BIS & $(7.7 \pm 2.2) \times 10^{6}$ & $(8.2 \pm 0.4) \times 10^{6}$ & 94 \\
ULC & $(4.1 \pm 0.3) \times 10^{6}$ & $(6.9 \pm 0.3) \times 10^{6}$ & 60 \\
\hline
\end{tabular}

The volumes of the $5 \mathrm{~mol} \%$ BIS and ULC microgels adsorbed to a PAH-coated substrate via spin-coating and studied in the dry state were determined as described in the Experimental Section and are listed in Table S3. In addition they were compared to the microgels volume in bulk solution in the collapsed state, since it is the state of the microgel in which it is containing the least water. The volume of the 5 mol\% BIS microgels is reduced by the dehydration to only $60 \%$ of the volume in bulk. In case of the ULC microgels, both volumes are nearly identical. It has to be mentioned that the volume of the ULC microgels in dry state is having a huge error.

Table S3. Volumes obtained by AFM height images $V_{A F M}$ of the microgels adsorbed to a PAH-coated glass substrate via spincoating in dry state, volumes obtained by DLS $V_{D L S}$, and ratio between the two $V_{A F M} / V_{D L S}$. Volumes $V_{A F M}$ were extracted as described in the Experimental Section in case of the 5 mol\% BIS microgels (with an addition of a volume of a cylinder with the total contact radius and a height of $1 \mathrm{~nm}$ for the polymeric thin layer around the protruding densely cross-linked part), and in case of ULC microgels the volume of a cylinder with the determined contact radius and a height of $1.5 \mathrm{~nm}$ was calculated. Volumes $V_{D L S}$ of the microgels in bulk were calculated using hydrodynamic radii $R_{h}$ at $T=40^{\circ} \mathrm{C}$ as a radius of a sphere.

\begin{tabular}{lccc}
\hline Sample type & $\begin{array}{c}\mathbf{V}_{\text {AFM, dry }} \\
{\left[\mathbf{n m}^{\mathbf{3}}\right]}\end{array}$ & $\begin{array}{c}\mathbf{V}_{\text {DLS, 40 }} \mathbf{C}^{\mathbf{C}} \\
{\left[\mathbf{n m}^{\mathbf{3}}\right]}\end{array}$ & $\begin{array}{c}\mathbf{V}_{\text {AFM, dry }} / \mathbf{V}_{\mathbf{D L S}, \mathbf{4 0}^{\circ} \mathbf{C}} \\
{[\%]}\end{array}$ \\
\hline $5 \mathrm{~mol} \%$ BIS & $(5.1 \pm 0.4) \times 10^{6}$ & $(8.2 \pm 0.4) \times 10^{6}$ & 63 \\
ULC & $(7.6 \pm 2.8) \times 10^{6}$ & $(6.9 \pm 0.3) \times 10^{6}$ & 110 \\
\hline
\end{tabular}




\section{IMPACT OF APPLIED FORCE DURING IMAGING}

The impact of the applied force during imaging on the microgels' dimensions was quantified by force-distance curves acquired at the apex of the 5 mol\% BIS (A) and ULC microgels $(\mathbf{B})$ at $T=35^{\circ} \mathrm{C}$ after in situ adsorption in comparison to curves acquired on the clean substrate. The position of the nanoindentation was determined by previous images. The force-distance curves were first-order baseline-corrected. The distance, at which the force started to increase above the noise level (ca. $50 \mathrm{pN}$ ), was assigned to the contact-point of probe and sample. Corrected force-distance curves were obtained by subtraction of the contact-point distance from the raw data.
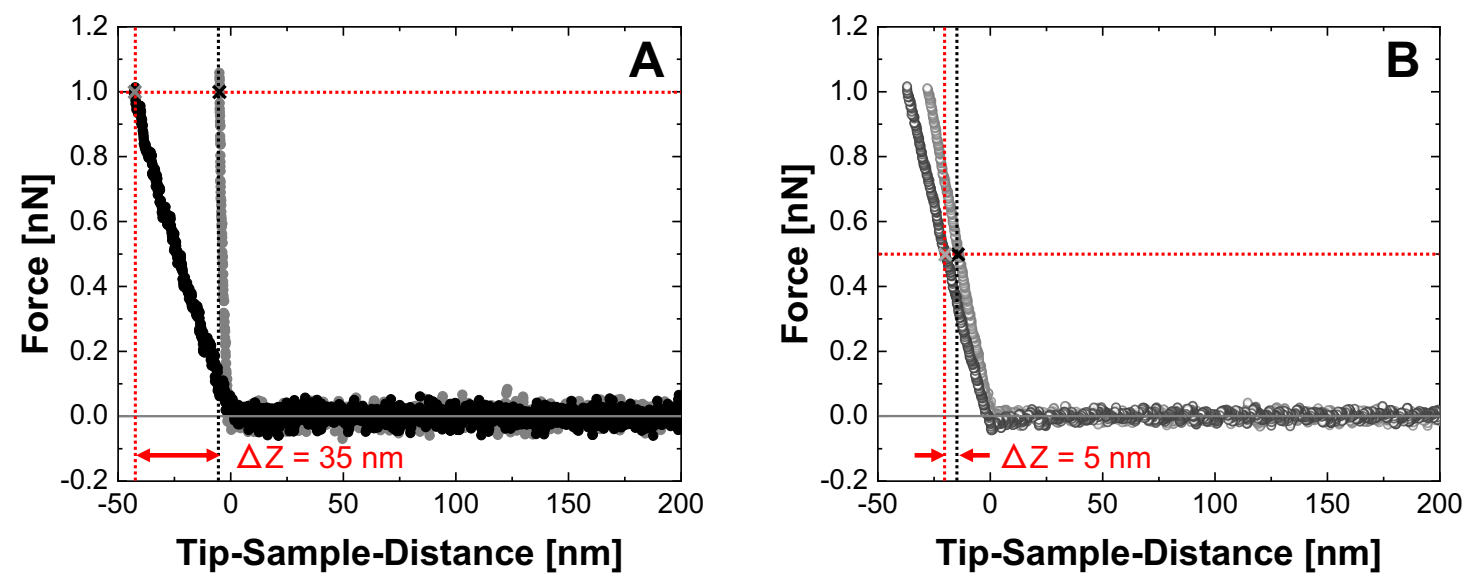

Figure S5. Force-distance curves at the apex of the in situ adsorbed 5 mol\% BIS (A, filled black circles) and ULC (B, open dark gray circles) microgels at $T=35{ }^{\circ} \mathrm{C}$. The impact of the applied force during imaging is quantified by the comparison to the indentation on the clean solid substrate (light gray, filled circles in A and open circles in B). A: 5 mol\% BIS microgels were imaged with a force of $F=1.0 \mathrm{nN}$ resulting in a deformation by $\Delta Z=35 \mathrm{~nm}$. B: ULC microgels were imaged with a force of $F=0.5 \mathrm{nN}$ resulting in a deformation by $\Delta Z=5 \mathrm{~nm}$.

The $5 \mathrm{~mol} \%$ BIS microgels were imaged with an applied force of $F=1.0 \mathrm{nN}$. In comparison to the indentation on the clean solid substrate this results in a deformation by $\Delta Z=35 \mathrm{~nm}$, which corresponds to less than $15 \%$ of the total height. The impact of the force applied during imaging is less for the ULC microgels. The ULC microgels were imaged with an applied force of $F=0.5 \mathrm{nN}$. The resulting deformation is only $\Delta Z=5 \mathrm{~nm}$, corresponding to less than $5 \%$ of the total height.

[1] M. Stieger, W. Richtering, J. S. Pedersen and P. Lindner, J. Chem. Phys., 2004, 120, 6197-206.

[2] M. F. Schulte, A. Scotti, A. P. H. Gelissen, W. Richtering and A. Mourran, Langmuir, 2018, 34, 4150-4158.

[3] J. C. Crocker and D. G. Grier, J. Colloid Interface Sci., 1996, 179, 298-310. 